\title{
DISEÑO E IMPLEMENTACIÓN DE UNA RED NEURONAL ARTIFICIAL PARA PREDECIR EL RENDIMIENTO ACADÉMICO EN ESTUDIANTES DE INGENIERÍA CIVIL DE LA UNIFSLB
}

\section{DESIGN AND IMPLEMENTATION OF AN ARTIFICIAL NEURAL NETWORK TO PREDICT ACADEMIC PERFORMANCE IN CIVIL ENGINEERING STUDENTS FROM UNIFSLB}

\author{
Fernando Alain Incio Flores ${ }^{1}$ \\ https://orcid.org/0000-0003-3286-7787 \\ fincio@ucvvirtual.edu.pe \\ Dulce Lucero Capuñay Sanchez ${ }^{1}$ \\ https://orcid.org/0000-0001-8678-5766 \\ dcapunay@ucvvirtual.edu.pe \\ Ronald Omar Estela Urbina ${ }^{2}$ \\ https://orcid.org/0000-0001-5240-1242 \\ restela@unibagua.edu.pe \\ Jorge Antonio Delgado Soto ${ }^{3}$ \\ https://orcid.org/0000-0003-2275-8608 \\ jdelgado@unc.edu.pe \\ Segundo Edilberto Vergara Medrano ${ }^{4}$ \\ (iD https://orcid.org/0000-0001-7611-3827 \\ svergara@unj.edu.pe \\ Aceptado: $20 / 03 / 2021$ \\ Publicado online:30/05/2021
}

\begin{abstract}
RESUMEN
Predecir los resultados académicos de los estudiantes permite al docente buscar técnicas y estrategias en el tiempo indicado durante el proceso de enseñanza y aprendizaje con el fin de mejorar el logro de competencias en sus estudiantes. En esta investigación se implementó una red neuronal artificial (RNA) para predecir los resultados académicos del curso de física de los estudiantes del II ciclo de la carrera profesional de Ingeniería Civil de la universidad Nacional Intercultural Fabiola Salazar Leguía de Bagua-Perú en función de datos históricos. La RNA se diseñó e implemento en el Software MATLAB, su arquitectura está formada por una capa de entrada, una capa oculta y una capa de salida, para el entrenamiento de la RNA se utilizó dos algoritmos que posee la Toolbox de MATLAB: el Scaled Conjugate Gradient logrando un porcentaje de predicción del $70 \%$ y el Levenberg-Marquardt logrando un porcentaje de predicción $86 \%$.
\end{abstract}

Palabras clave: Rendimiento académico, red neuronal artificial, predicción.

\footnotetext{
1 Universidad Cesar Vallejo. Escuela de Postgrado. Perú.

2 Universidad Nacional Intercultural Fabiola Salazar Leguía de Bagua. Perú.

${ }^{3}$ Universidad Nacional de Cajamarca. Facultad de Educación. Perú.

${ }^{4}$ Universidad Nacional de Jaén. Facultad de Ingeniería Forestal y Ambiental. Perú.
} 


\begin{abstract}
Predicting the academic results of students allows the teacher to seek techniques and strategies at the indicated time during the teaching and learning process in order to improve the achievement of skills in their students. In this research, an artificial neural network (ANN) was implemented to predict the academic results of the physics course of the students of the II cycle of the Civil Engineering career of the National Intercultural University Fabiola Salazar Leguía de Bagua-Peru based on data historical. The RNA was designed and implemented in the MATLAB Software, its architecture is made up of an input layer, a hidden layer and an output layer, for the RNA training two algorithms that the MATLAB Toolbox has: the Scaled Conjugate Gradient achieving a prediction percentage of $70 \%$ and the Levenberg-Marquardt achieving a prediction percentage of $86 \%$.
\end{abstract}

Keywords: Academic performance, artificial neural network, prediction.

\title{
INTRODUCCIÓN
}

Las redes neuronales artificiales a lo largo de los años se han convertido en modelos efectivos de predicción, han demostrado mejor efectividad frente a modelos estadísticos de regresión sin necesidad de cumplir condiciones de linealidad, normalidad, tamaño muestral, entre otros (ArandaCorral et al., 2014; Samani et al., 2017). La Matemática es el sustento de la Física, y la Física es el sustento de la ingeniería (Popenici y Kerr, 2017), los datos proporcionados por la oficina de asuntos académicos de la Universidad Nacional Intercultural Fabiola Salazar Leguía de Bagua (UNIFSLB) evidencian cierto grado de dificultad de los estudiantes de Ingeniería Civil para el logro de competencias en la asignatura de Física, evidentemente esto origina preocupación puesto que la carrera profesional de Ingenería Civil tiene un fundamento físico-matemático, en tal sentido surge la necesidad de implementar modelos matemáticos computacionales que permitan predecir los resultados académicos haciendo uso de los datos históricos con el objetivo de proporcionar al docente información oportuna y a tiempo para que pueda hacer uso de diversas técnicas y estrategias de aprendizaje a fin de mejorar estos posibles resultados.

Una RNA es un modelo matemático computacional formado por una capa de entrada, capas de salidas y capas ocultas, estás a su vez están constituidas por neuronas artificiales e interconectadas mediante sinapsis, cada sinapsis con su respectivo peso, el funcionamiento de una RNA se asemeja al comportamiento del cerebro humano, capaz de aprender mediante el aprendizaje, precedido de un entrenamiento repetitivo (Amershi et al., 2005; Brazdil y Jorge, 2001; Brusilovsky y Peylo, 2003; Garrido, 2010; Vila y Penín, 2007).

La educación universitaria en el siglo XXI se ha caracterizado por fomentar el logro de competencias en los estudiantes, en este sentido el rendimiento académico es la evaluación cuantitativa y cualitativa del logro de competencias expresados finalmente en una escala vigesimal en el sistema de evaluación de la UNIFSLB. El logro o no de las competencias en los estudiantes está influenciado por diversos factores de orden emocional, cognitivo y socio cultural (Cerda et al., 2019; Edel, 2003; Garbanzo, 2007; Pinto et al., 2020; Salanova et al., 2005) quienes intervienen positivamente o negativamente en el rendimiento académico de los estudiantes.

Las RNAs es una de las herramientas que ofrece la inteligencia artificial (IA), muy potente y efectiva para el pronóstico de eventos y fenómenos, a lo largo de las décadas se ha evidenciado un incremento significativo de investigaciones en el campo de la educación que involucren redes neuronales artificiales como herramienta para dar solución a problemas que acechan la formación académica de los estudiantes. Es así como en la década del 70 se inician las primeras investigaciones que dan origen a programadas de tutorías y aprendizajes automatizados mediante redes neuronales y sistemas expertos orientados al seguimiento y evaluación de estudiantes y docentes, los resultados no 
fueron los esperados por los investigadores, pero significó el punto de partida para investigaciones posteriormente exitosas (John Seely Brown y Burton, 1978; J.S. Brown y Burton, 1978; Gable y Page, 1980; Goldstein y Papert, 1977; Self, 1979). La década de los 80 y 90 se caracterizó por el perfeccionamiento y adaptación de las diversas herramientas de la IA en investigaciones dentro del campo de la educación, la aplicación de los sistemas de tutoría automatizados y los resultados en la predicción mejoraron considerablemente como consecuencia de perfeccionamiento e implementación de nuevos algoritmos computacionales (Boden, 1984; Salchenberger, 1989; Schaufelberger, 1993; Siemer y Angelides, 1998; Wasson, 1997).

En este nuevo milenio las investigaciones en educación utilizando RNA a tomado notoriedad, entre ellas podemos mencionar la realizada por Çetinkaya y Baykan (2020) en Rusia, en la que implementaron un modelo de RNA para pronosticar el talento de los estudiantes en programación utilizando tres algoritmos computacionales implementados en el MATLAB, así mismo Álvarez et al. (2016) en Cuba mediante una RNA pronosticaron el rendimiento académico de los estudiantes correspondientes al curso de Estructura de Datos I y II, en la república de Chile Zambrano et al. (2011) mediante RNA y Data Warehouse pronosticó el rendimiento académico de los estudiantes de Ingeniería Civil de la Universidad de Atacama utilizando datos históricos.

Esta investigación se justifica en la necesidad de diseñar e implementar un modelo de red neuronal artificial que permita predecir los resultados académicos de los estudiantes antes de culminar el semestre académico, con la finalidad de que el docente utilicé técnicas y estrategias de aprendizaje adecuadas para mejorar los posibles resultados académicos obtenidos por sus estudiantes.

\section{METODOLOGÍA}

Los datos que alimentaron la capa de entrada de la RNA se obtuvieron mediante la técnica de la encuesta aplicada a 50 estudiantes de la carrera profesional de Ingeniería Civil que ya aprobaron la asignatura de Física, se utilizó como instrumento un cuestionario con un coeficiente de confiabilidad de Alpha de Cronbach de 0.854 , las preguntas que conforman el cuestionario son 18 y responden a los factores de orden cognitivo (9), emocional (6) y socio cultural (3) que influyen en el rendimiento académico de los estudiantes. Las 18 preguntas recolectan información referida a planes de estudios, nivel educativo de los padres, servicios de internet, características demográficas, calificaciones obtenidas en el primer ciclo en asignaturas que son pre requisitos para Física, calificaciones obtenidas en la secundaria, calificación obtenida en el examen de admisión, el autoconcepto académico, la vocación, la motivación, sexo, edad, estado civil, carga familiar, consumo de drogas, consumo de alcohol.

Se utilizó el diseño metodológico CRIS-DM, primero se identificó el problema y las variables de estudio, se elaboró el instrumento de recolección de datos y se procedió a la recolección, selección y preparación de los mismos, luego se diseñó la arquitectura de la red neuronal artificial, se validó el modelo de RNA implementado tomando un porcentaje de los datos para el entrenamiento, la validación y la prueba utilizando los algoritmos Scaled Conjugate Gradient y Levenberg-Marquardt, los algoritmos se ejecutaron 10 veces por cada número de neuronas en la capa oculta, el número de neuronas en la capa oculta variaba entre 2 y 50 neuronas, se trataba de buscar que el error cuadrático medio (MSE) sea lo más cercano a cero y el coeficiente de correlación (R) sea lo más cercano a uno, siendo:

$$
M S E=\frac{1}{n} \sum_{i=1}^{n}\left(y_{i c}-y_{i m}\right)^{2}
$$




$$
R^{2}=1-\frac{\sum_{i=1}^{n}\left(y_{i c}-y_{i m}\right)^{2}}{\sum_{i=1}^{n}\left(y_{i c}-y_{m}\right)^{2}}
$$

Donde " $n$ " es el número de observaciones, yic es el i-ésimo valor calculado, yim es el i-ésimo valor medido, finalmente $y_{m}$ es el valor medio de los valores calculados.

\section{Figura 1}

Diagrama secuencial del diseño metodológico

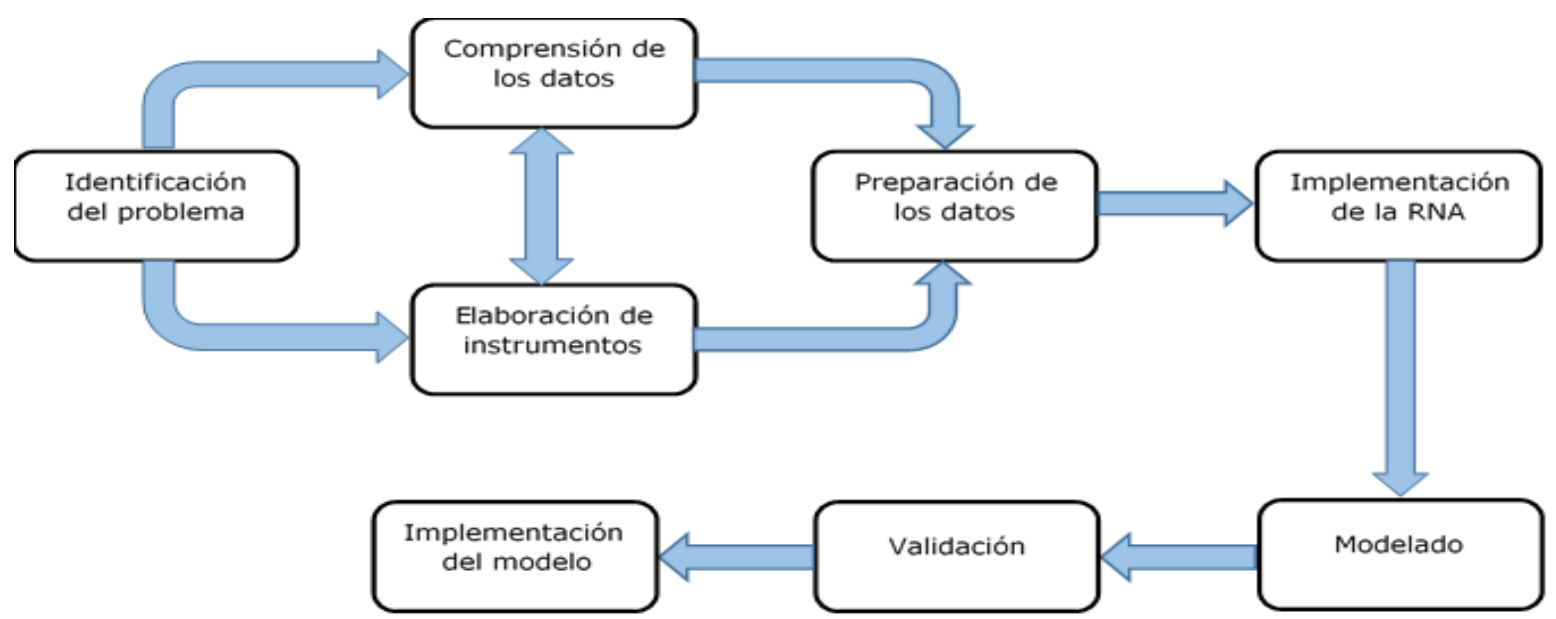

\section{RESULTADOS Y DISCUSIÓN}

Los mejores resultados en la implementación de la red neuronal se obtuvieron al emplear el 85\% de los datos para el entrenamiento (training), el 10\% para la validación (validation) y el 5\% para la prueba (test), se efectúo 10 corridas para cada algoritmo de aprendizaje, con 14 neuronas en la capa oculta para el Levenberg-Marquardt y 50 neuronas en la capa oculta para el Scaled Conjugate Gradient. La Tabla 1 detalla los valores obtenidos en el MSE y en el $\mathrm{R}$ de las redes neuronales con respecto a cada algoritmo de aprendizaje y al número de neuronas en la capa oculta ( $\mathrm{N}^{\circ} \mathrm{nco}$ ), la Figura 2 y la Figura 3 muestran los diagramas de las redes neuronales implementadas para cada algoritmo, la Figura 4 y la Figura 5 muestran el R-Training, R-Validation y el R-Test de ambos algoritmos, la Figura 6 y la Figura 7 muestran el histograma de error para los dos algoritmos, la Figura 8 y la Figura 9 muestran el rendimiento de ambos algoritmos.

\section{Tabla 1}

Análisis de los resultados

\begin{tabular}{|c|c|c|c|c|c|c|c|c|}
\hline Algoritmo & $\begin{array}{l}\mathrm{N}^{\circ} \\
\mathrm{nco}\end{array}$ & $\begin{array}{l}\text { Entrena- } \\
\text { miento } \\
\text { (Training-R) }\end{array}$ & $\begin{array}{l}\text { Validación } \\
\text { (Validatio } \\
n-R \text { ) }\end{array}$ & $\begin{array}{l}\text { Prueba } \\
\text { (Test-R) }\end{array}$ & All-R & $\begin{array}{l}\text { Entrenamiento } \\
\text { (Training -MSE) }\end{array}$ & $\begin{array}{l}\text { Validation } \\
\text { (Validation- } \\
\text { MSE) }\end{array}$ & $\begin{array}{l}\text { Prueba } \\
\text { (Test-MSE) }\end{array}$ \\
\hline $\begin{array}{l}\text { Levenberg- } \\
\text { Marquardt } \\
\text { Scaled }\end{array}$ & 14 & 0.99994 & 0.87178 & 0.95254 & 0.96723 & 0.00000389003 & 0.00264181 & 0.0138162 \\
\hline $\begin{array}{l}\text { Conjugate } \\
\text { Gradient }\end{array}$ & 50 & 0.98114 & 0.94263 & 0.759620 & 0.76359 & 0.00043774 & 0.0951 & 0.0990 \\
\hline
\end{tabular}

Fuente: elaboración propia 
Figura 2

Diagrama de la red neuronal- Levenberg-Marquardt

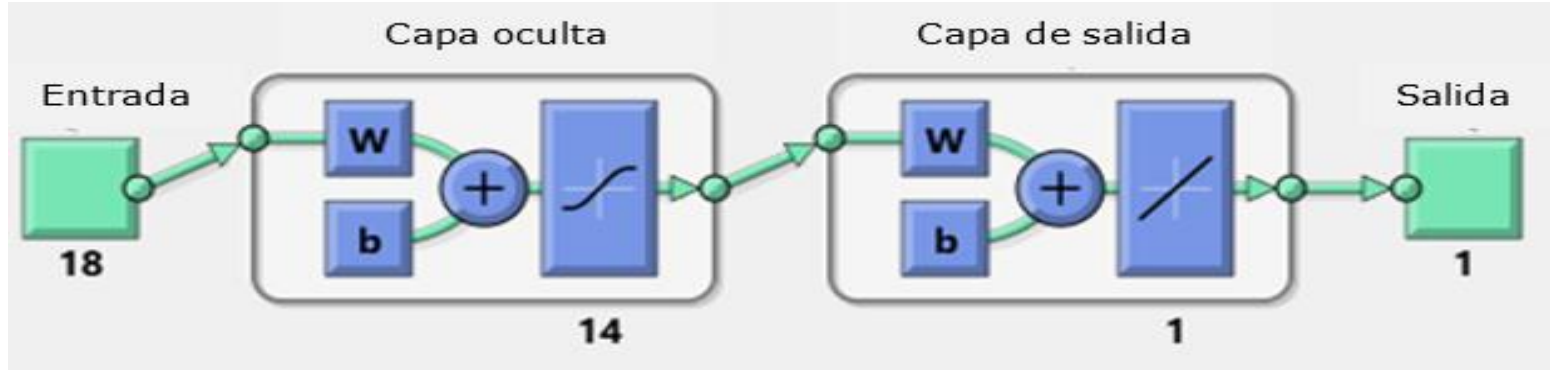

Nota. Red neuronal artificial implementada indicando el número de neuronas en cada capa.

Figura 3

Diagrama de la red neuronal-Scaled Conjugate Gradient

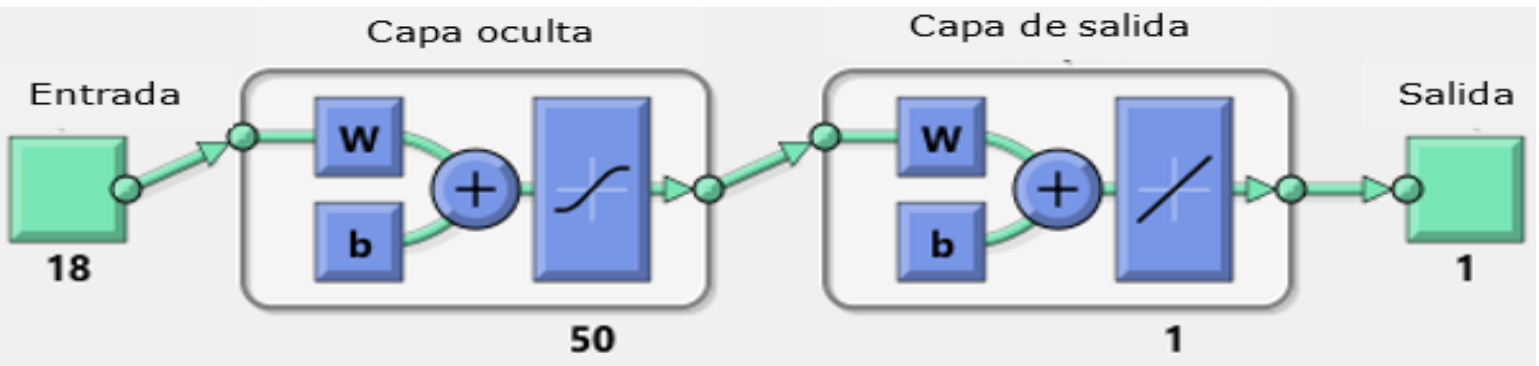

Nota. Red neuronal artificial implementada indicando el número de neuronas en cada capa.

Figura 4

$R$ del Levenberg-Marquardt
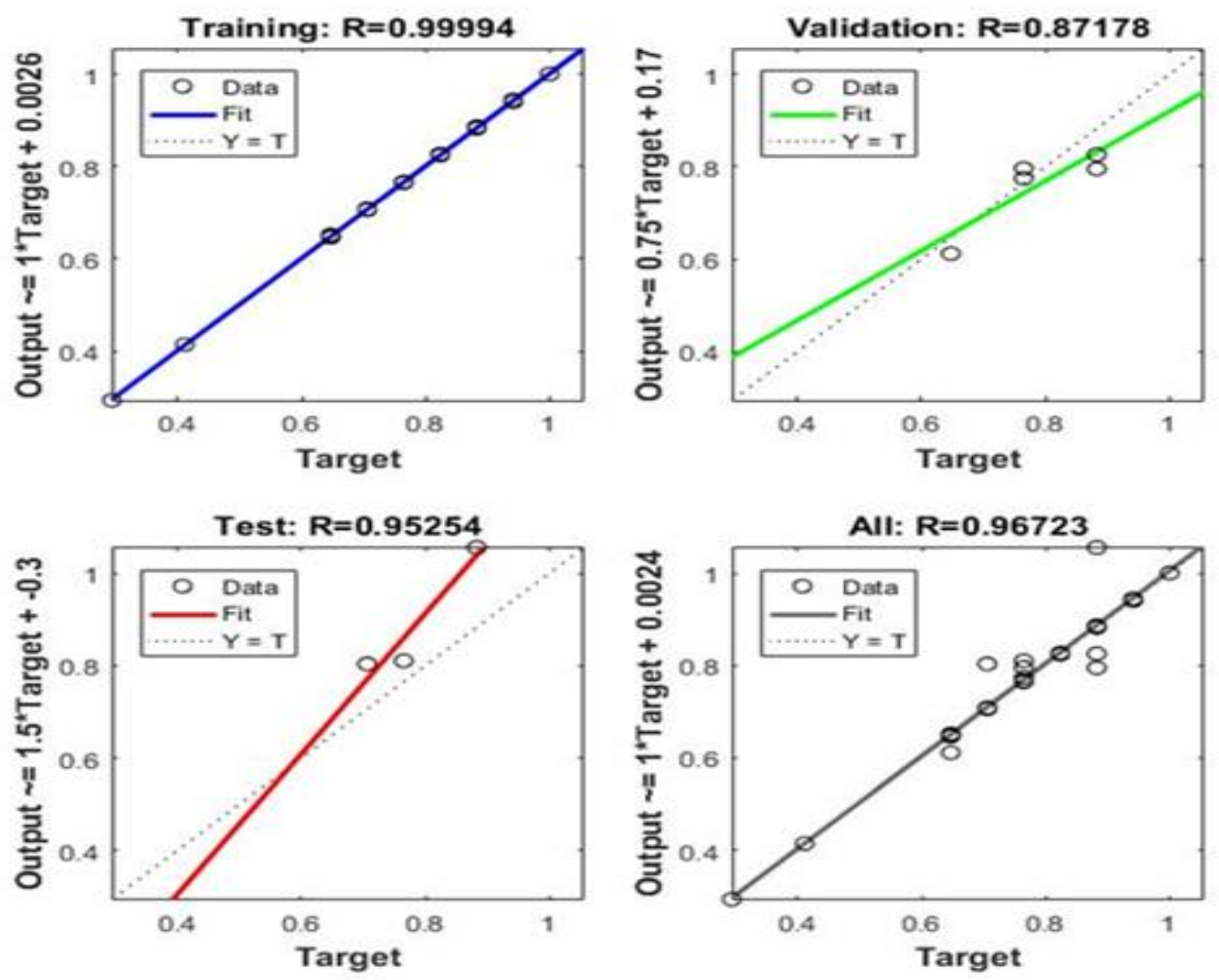
Figura 5

$R$ del Scaled Conjugate Gradient.
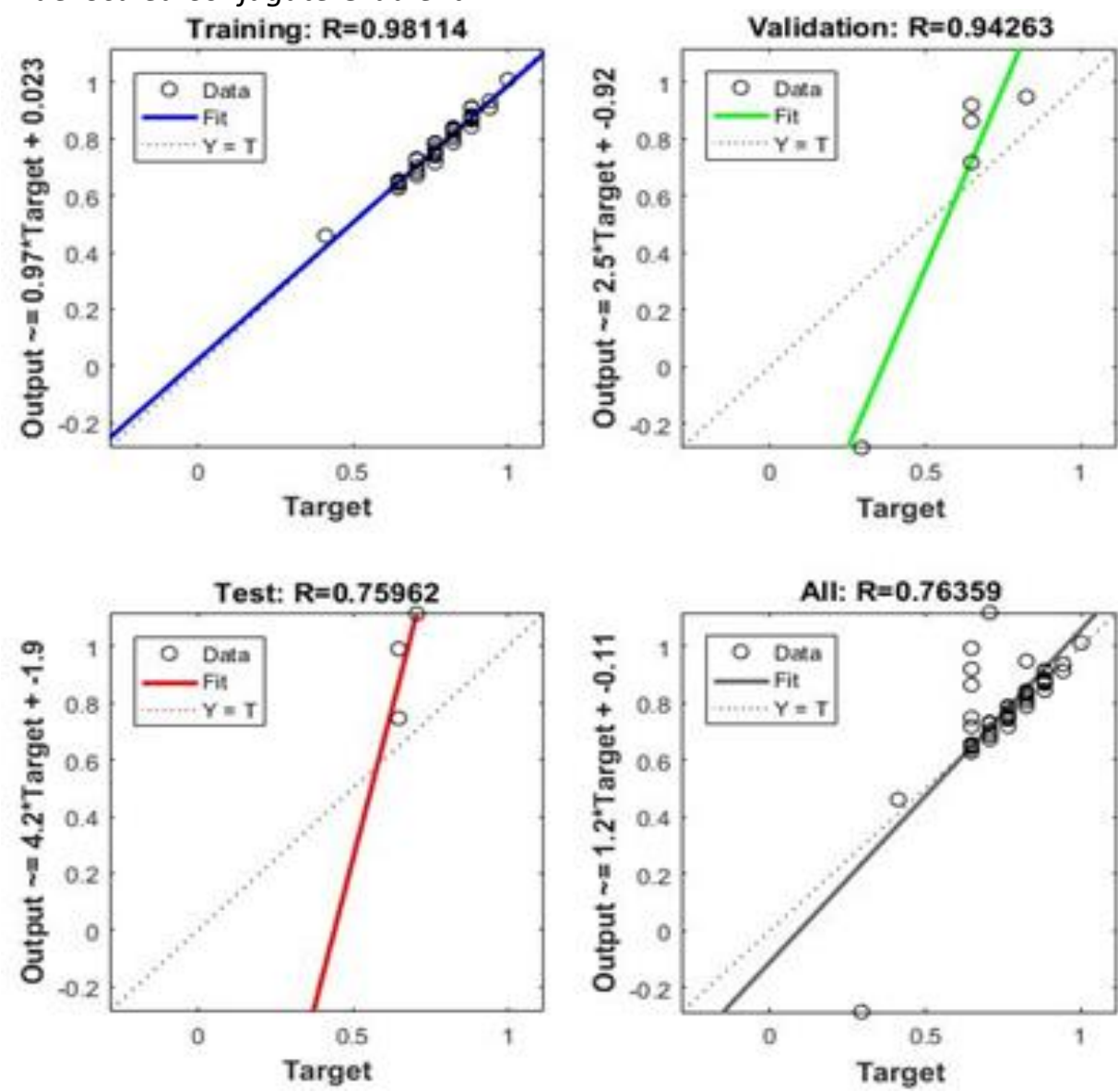

Figura 6

Histograma de error del Levenberg-Marquardt

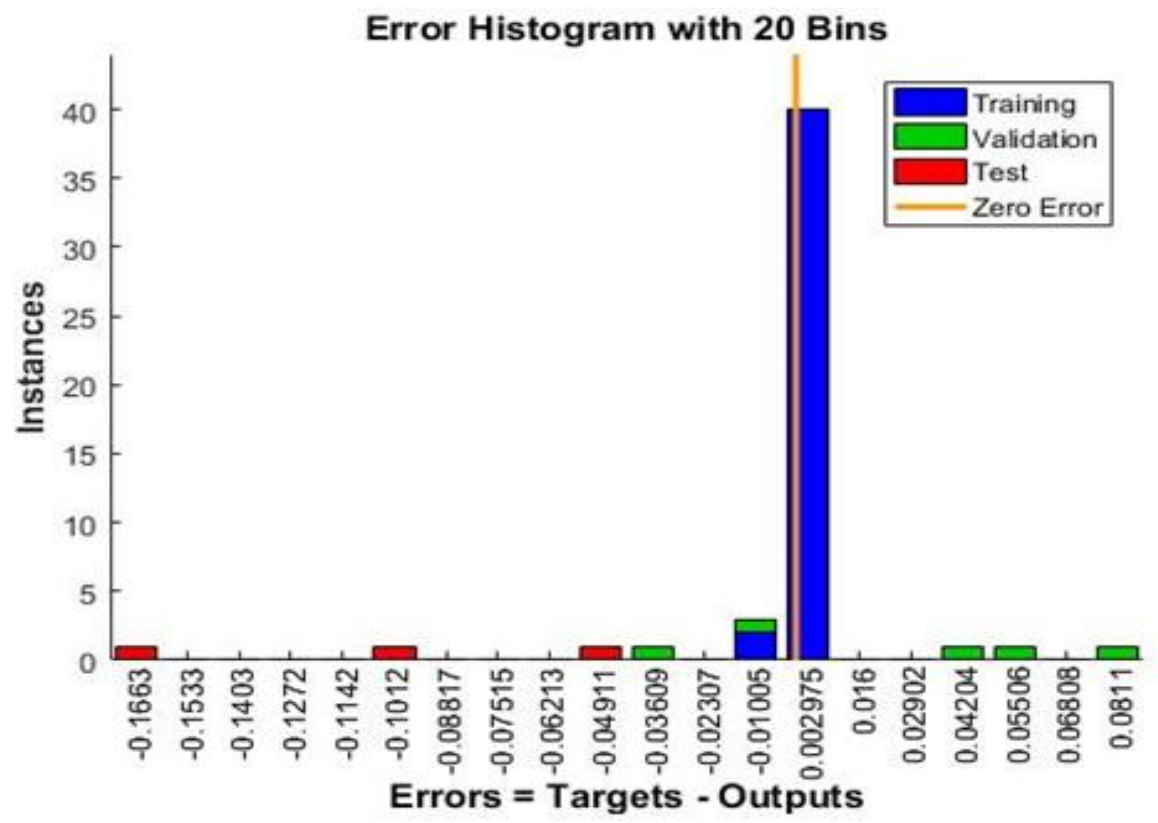




\section{Figura 7}

Histograma de error del Scaled Conjugate Gradient

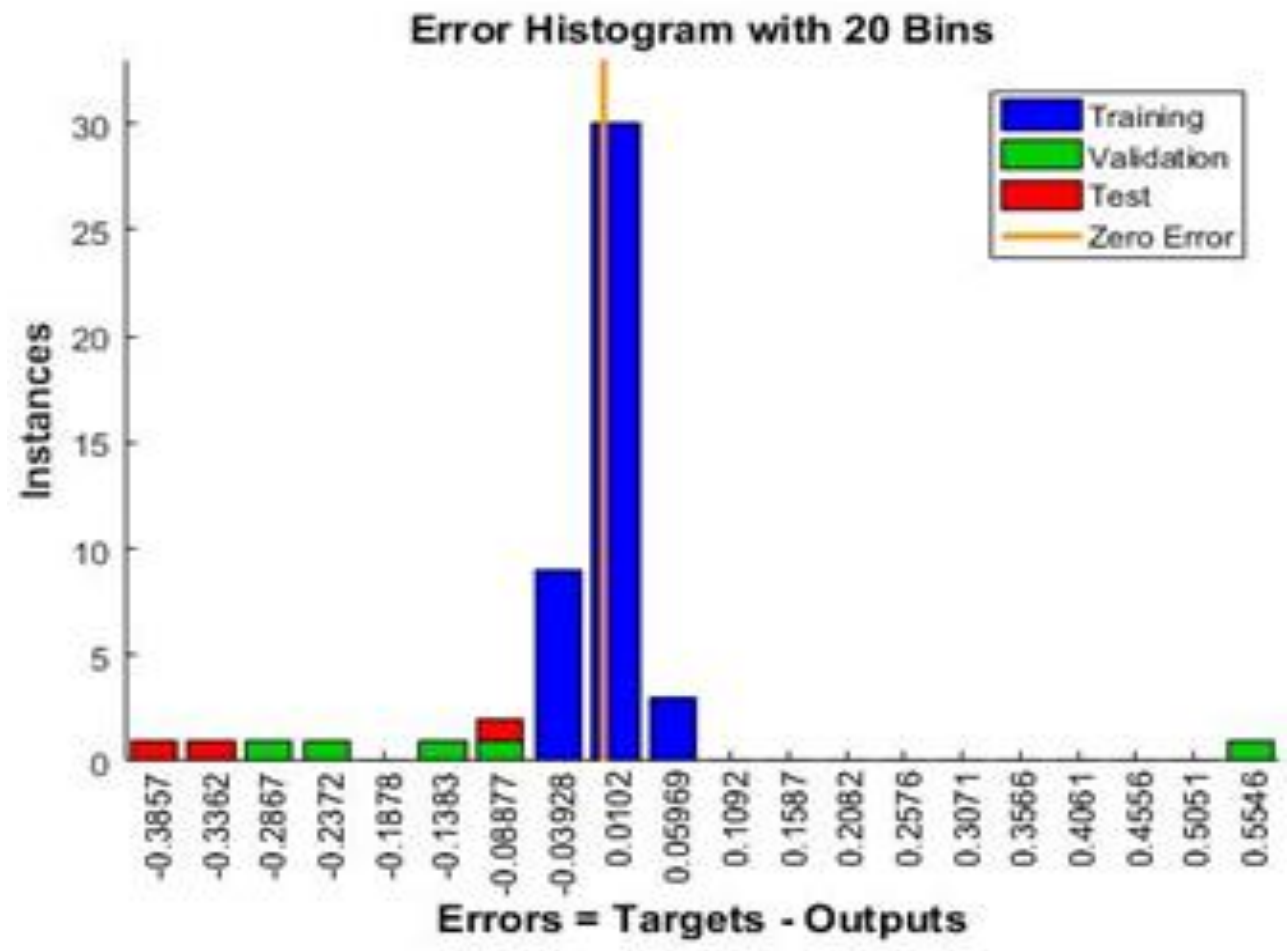

Figura 8

Rendimiento del Levenberg-Marquardt

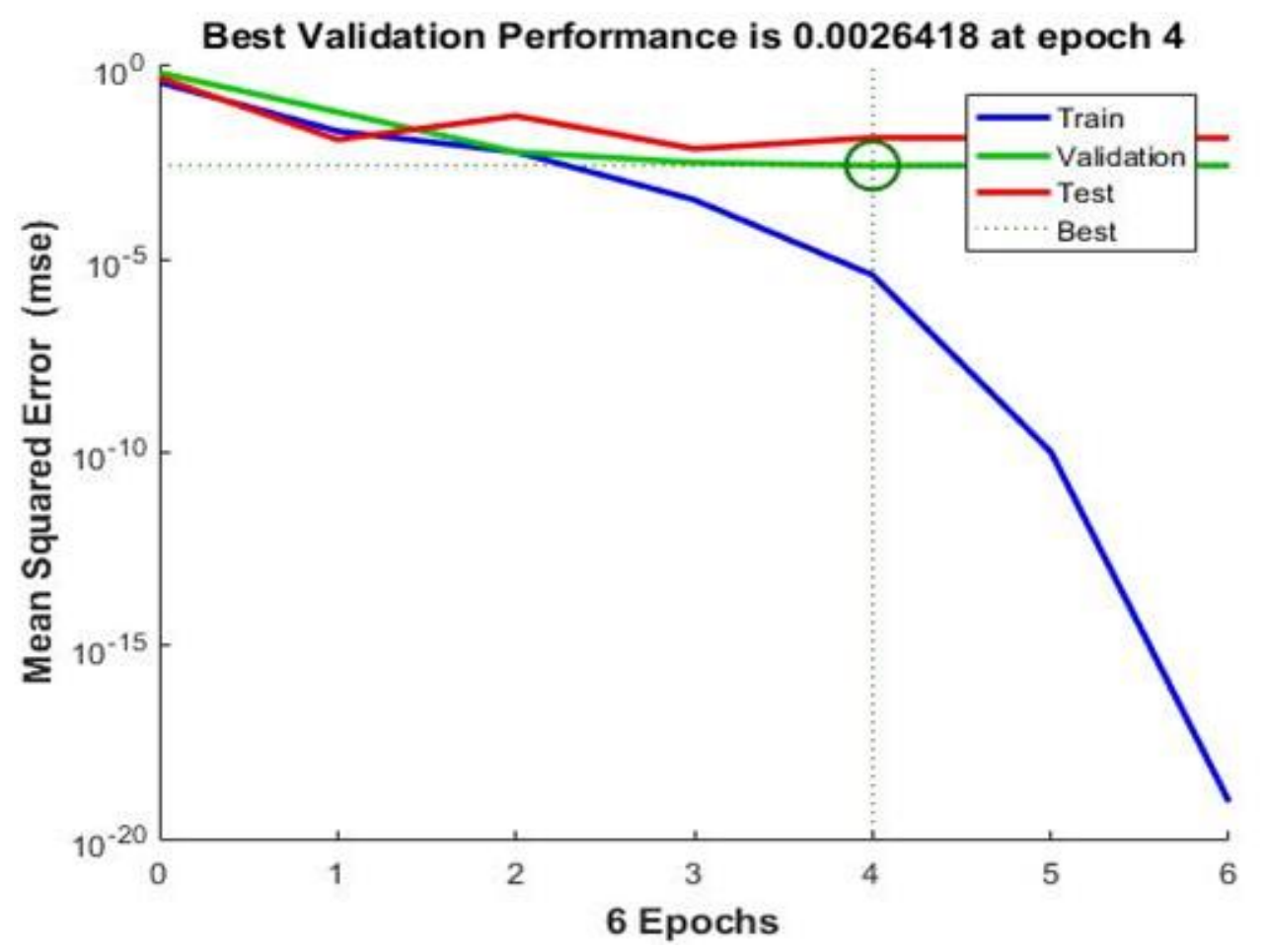


Figura 9

Rendimiento del Scaled Conjugate Gradient.

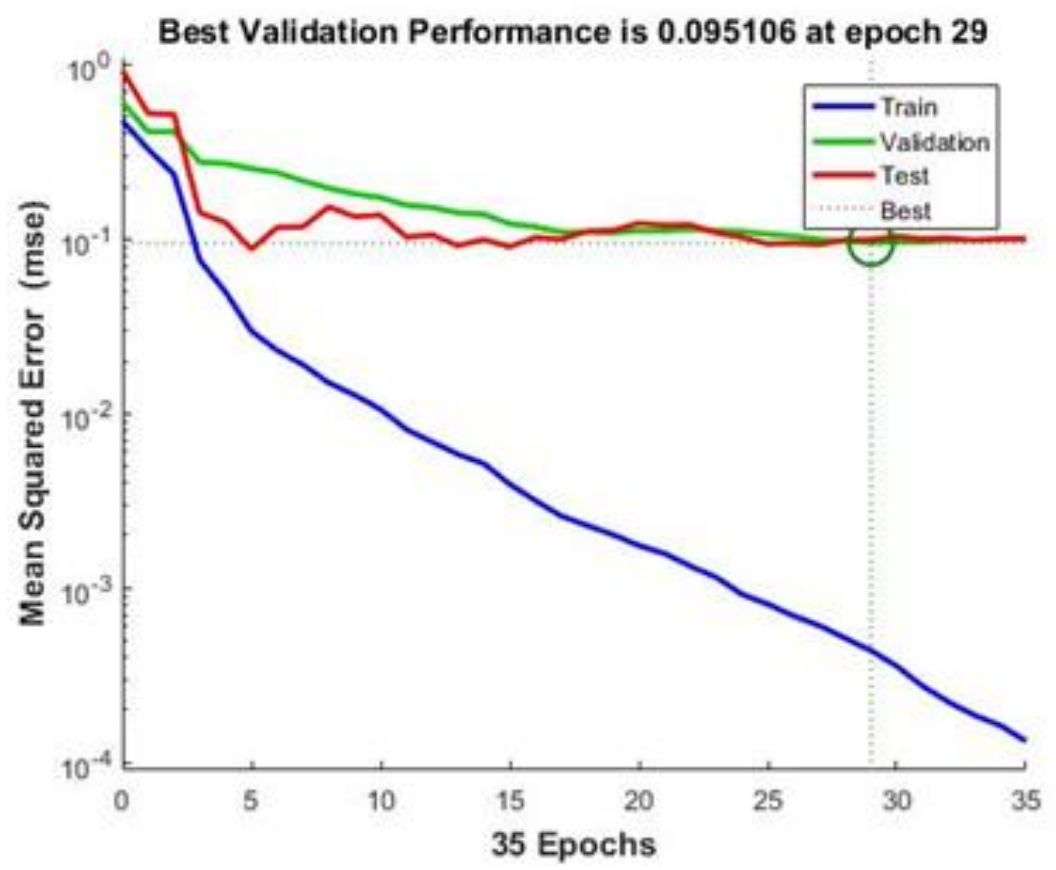

Tabla 2

Pronóstico de promedios finales

\begin{tabular}{|c|c|c|}
\hline \multirow{2}{*}{$\begin{array}{l}\text { Promedios } \\
\text { finales reales }\end{array}$} & \multicolumn{2}{|c|}{ Pronóstico } \\
\hline & LM & SCG \\
\hline 11 & 11 & 17 \\
\hline 13 & 13 & 12 \\
\hline 15 & 15 & 15 \\
\hline 14 & 14 & 14 \\
\hline 11 & 11 & 11 \\
\hline 15 & 15 & 14 \\
\hline 13 & 13 & 13 \\
\hline 14 & 14 & 13 \\
\hline 13 & 13 & 13 \\
\hline 17 & 17 & 17 \\
\hline 12 & 12 & 12 \\
\hline 15 & 14 & 15 \\
\hline 13 & 13 & 13 \\
\hline 15 & 14 & 15 \\
\hline 13 & 13 & 13 \\
\hline 16 & 16 & 15 \\
\hline 16 & 16 & 16 \\
\hline 15 & 15 & 15 \\
\hline 13 & 14 & 13 \\
\hline 12 & 12 & 11 \\
\hline 14 & 14 & 14 \\
\hline 12 & 14 & 12 \\
\hline 15 & 15 & 15 \\
\hline 13 & 13 & 13 \\
\hline 11 & 11 & 11 \\
\hline 14 & 14 & 14 \\
\hline
\end{tabular}

\begin{tabular}{|c|c|c|}
\hline 14 & 14 & 14 \\
\hline 11 & 11 & 11 \\
\hline 14 & 14 & 16 \\
\hline 11 & 11 & 16 \\
\hline 11 & 11 & 15 \\
\hline 12 & 12 & 19 \\
\hline 12 & 12 & 12 \\
\hline 15 & 15 & 15 \\
\hline 13 & 13 & 13 \\
\hline 14 & 14 & 14 \\
\hline 11 & 11 & 12 \\
\hline 11 & 11 & 13 \\
\hline 14 & 14 & 14 \\
\hline 13 & 14 & 13 \\
\hline 5 & 5 & -5 \\
\hline 11 & 11 & 11 \\
\hline 11 & 10 & 11 \\
\hline 7 & 7 & 8 \\
\hline 12 & 12 & 12 \\
\hline 15 & 18 & 16 \\
\hline 13 & 13 & 13 \\
\hline 12 & 12 & 12 \\
\hline 12 & 12 & 12 \\
\hline 11 & 11 & 11 \\
\hline$\%$ del & $86 \%$ & $70 \%$ \\
\hline pronóstico & & \\
\hline
\end{tabular}

Fuente: elaboración propia 
En la Tabla 1 se puede observar que, de los dos algoritmos, el Levenberg-Marquardt con 14 neuronas da mejores resultados en la predicción en menor tiempo tal como lo afirma Hagan y Menhaj (1994). Se puede observar: Training- $R=0.99994$, Validation $-R=0.87178$, Test- $R=0.95254$, All- $R=$ 0.96723 , Training-MSE $=0.00000389003$, Validation-MSE $=0.00264181$, Test-MSE $=0.0138162$.

En el histograma de error se simboliza el entrenamiento de los datos, las barras verdes representan la validación de los mismos y las barras rojas la prueba de los datos, en el caso del algoritmo Levenberg-Marquardt la mayoría de los errores se encuentra entre -0.1012 y 0.05506 , se pueden observar un valor atípico en -0.1663 y en 0.0811 , en el caso del algoritmo Scaled Conjugate Gradient la mayoría de los errores se encuentran ubicados entre -0.3857 y 0.05969 , se puede observar un valor atípico en 0.5546. La Tabla 2 muestra que la red entrenada por medio del algoritmo Levenberg-Marquardt (LM) tiene una capacidad de predicción del $86 \%$, y la red entrenada por medio del algoritmo Scaled Conjugate Gradient (SCG) tiene una capacidad de predicción del 70\%.

\section{CONCLUSIONES}

El modelo de red neuronal artificial implementada en esta investigación no debe ser tomada como válida en otra universidad puesto que las características de orden cognitivo, emocional y socio cultural es propio de cada población y varía de acuerdo a las condiciones de espacio-tiempo. No obstante, la metodología, antecedentes de estudio y los criterios para el diseño de la arquitectura de la RNA pueden considerarse para investigaciones dentro del contexto local, regional, nacional e internacional.

Se ha demostrado que las RNA constituyen una herramienta poderosa de predicción, la red entrenada por medio del algoritmo Levenberg-Marquardt (LM) tiene una capacidad de predicción del $86 \%$, y la red entrenada por medio del algoritmo Scaled Conjugate Gradient (SCG) tiene una capacidad de predicción del $70 \%$, lo cual resulta beneficioso para la comunidad estudiantil, pues le permite al docente conocer los posibles resultados académicos de sus estudiantes antes de culminar el semestre académico y de esta manera aplicar técnicas y estrategias a fin de mejorar el logro de competencias de sus estudiantes.

Se evidencia también que para lograr un alto porcentaje en la predicción es importante tener en cuenta que el coeficiente de confiabilidad del Alpha de Cronbach sea los más cercano a uno, que la red neuronal artificial implementada debe ser entrenada tantas veces sea necesario teniendo en cuenta el número de neuronas en la capa oculta y el algoritmo de entrenamiento. Una limitación en esta investigación es la cantidad de datos, no obstante, a quedado demostrado que la RNA implementada es capaz de proporcionar un alto índice de porcentaje de predicción.

\section{REFERENCIAS BIBLIOGRÁFICAS}

Álvarez, J., Lau, R., Pérez, S., y Leyva, E. C. (2016). Predicción de resultados académicos de estudiantes de informática mediante el uso de redes neuronales. Ingeniare. Revista chilena de ingeniería, 24(4), 715-727. https://doi.org/10.4067/S0718-33052016000400015

Amershi, S., Arksey, N., Carenini, G., Conati, C., Mackworth, A., Maclaren, H., y Poole, D. (2005). Designing Clspace: Pedagogy and usability in a learning environment for Al. Proceedings of the 10th Annual SIGCSE Conference on Innovation and Technology in Computer Science Education - ITiCSE '05, 178. https://doi.org/10.1145/1067445.1067495

Aranda-Corral, G. A., Calmet, J., y Martín-Mateos, F. J. (Eds.). (2014). Artificial Intelligence and Symbolic Computation (Vol. 8884). Springer International Publishing. https://doi.org/10.1007/978-3319-13770-4

Boden, M. A. (1984). Impacts of artificial intelligence. Futures, 16(1), 60-70. https://doi.org/10.1016/0016-3287(84)90007-7 
Brazdil, P., y Jorge, A. (Eds.). (2001). Progress in Artificial Intelligence: Knowledge Extraction, Multiagent Systems,Logic Programming, and Constraint Solving (Vol. 2258). Springer Berlin Heidelberg. https://doi.org/10.1007/3-540-45329-6

Brown, John Seely, y Burton, R. R. (1978). A paradigmatic example of an artificially intelligent instructional system. International Journal of Man-Machine Studies, 10(3), 323-339. https://doi.org/10.1016/S0020-7373(78)80050-9

Brown, J.S., y Burton, R. R. (1978). Diagnostic models for procedural bugs in basic mathematical skills. Cognitive Science, 2(2), 155-192. Scopus. https://doi.org/10.1016/S0364-0213(78)80004-4

Brusilovsky, P., y Peylo, C. (2003). Adaptive and intelligent web-based educational systems. International Journal of Artificial Intelligence in Education, 13(2-4), 159-172. Scopus. https://www.scopus.com/inward/record.uri?eid=2-s2.085013582692\& partner|D=40\& $m d 5=8 \mathrm{fd} 4 \mathrm{cfe} 2 \mathrm{~d} 1364 \mathrm{a} 872 \mathrm{~d} 3 \mathrm{~b} 599134 \mathrm{c} 1 \mathrm{f} 432$

Cerda, G., Pérez, C., Elipe, P., Casas, J. A., y Del Rey, R. (2019). Convivencia escolar y su relación con el rendimiento académico en alumnado de Educación Primaria. Revista de Psicodidáctica, 24(1), 46-52. https://doi.org/10.1016/j.psicod.2018.05.001

Çetinkaya, A., y Baykan, Ö. K. (2020). Prediction of middle school students' programming talent using artificial neural networks. Engineering Science and Technology, an International Journal. https://doi.org/10.1016/j.jestch.2020.07.005

Edel, R. (2003). Factores asociados al rendimiento académico. Revista Iberoamericana de Educación, 33(1), 1-20. https://doi.org/10.35362/rie3312872

Gable, A., y Page, C. V. (1980). The use of Artificial Intelligence techniques in Computer-Assisted Instruction: An overview. International Journal of Man-Machine Studies, 12(3), 259-282. https://doi.org/10.1016/S0020-7373(80)80028-9

Garbanzo, G. M. (2007). Factores asociados al rendimiento académico en estudiantes universitarios, una reflexión desde la calidad de la educación superior pública. Revista Educación, 31(1), 4363. https://www.redalyc.org/articulo.oa?id=44031103

Garrido, A. (2010). Mathematics and Artificial Intelligence, two branches of the same tree. Innovation and Creativity in Education, 2(2), 1133-1136. https://doi.org/10.1016/j.sbspro.2010.03.160

Goldstein, I., y Papert, S. (1977). Artificial intelligence, language, and the study of knowledge. Cognitive Science, 1(1), 84-123. https://doi.org/10.1016/S0364-0213(77)80006-2

Pinto, P. M., Fernández, Y. O., y Cabezas, N. G. (2020). Intercultural education in the process of educational humanization of college students: Influences on academic achievement. Interciencia, 45(4), 201-208. Scopus. https://www.scopus.com/inward/record.uri?eid=2-s2.085085116052\&partnerID $=40 \& \mathrm{md} 5=\mathrm{d} 5452 \mathrm{e} 892 \mathrm{f} 20 \mathrm{e} 1 \mathrm{~b} 42 \mathrm{dfe} 7 \mathrm{f} 487 \mathrm{dd} 4 \mathrm{ba} 51$

Popenici, S. A. D., y Kerr, S. (2017). Exploring the impact of artificial intelligence on teaching and learning in higher education. Research and Practice in Technology Enhanced Learning, 12(1). Scopus. https://doi.org/10.1186/s41039-017-0062-8

Salanova, M., Cifre, E., Grau, R. M., Llorens, S., y Martínez, I. M. (2005). Antecedentes de la autoeficacia en profesores y estudiantes universitarios: Un modelo causal. Revista de Psicología del Trabajo y de las Organizaciones, 21, 18.

Salchenberger, L. (1989). A strategy for integrating artificial intelligence technology into a graduate business curriculum. Education and Computing, 5(3), 189-196. https://doi.org/10.1016/S01679287(89)80042-1

Samani, T., Porayska-Pomsta, K., y Luckin, R. (2017). Bridging the gap between high and low performing pupils through performance learning online analysis and curricula: Vol. 10331 LNAI (p. 655). Scopus. https://doi.org/10.1007/978-3-319-61425-0_82

Schaufelberger, W. (1993). Low cost control education software for MS-DOS PCs. Automatica, 29(6), 1501-1516. https://doi.org/10.1016/0005-1098(93)90013-J

Self, J. A. (1979). Student models and artificial intelligence. Computers \& Education, 3(4), 309-312. https://doi.org/10.1016/0360-1315(79)90008-3 
Siemer, J., y Angelides, M. C. (1998). A comprehensive method for the evaluation of complete intelligent tutoring systems. Decision Support Systems, 22(1), 85-102. https://doi.org/10.1016/S0167-9236(97)00033-X

Vila, E. M. S., y Penín, M. L. (2007). Introduction to special issue Al techniches applied in education. $\begin{array}{llll}\text { Inteligencia } & \text { Artificial, } & \text { 71(33), } & \text { Scopus. }\end{array}$ https://www.scopus.com/inward/record.uri?eid=2-s2.036049016227\&partnerID=40\&md5=7a181a22f3b187c2e99100dbedea61e5

Wasson, B. (1997). Advanced educational technologies: The learning environment. Norwegian Perspectives on Computing in Complex Domains, 13(4), 571-594. https://doi.org/10.1016/S0747-5632(97)00027-7

Zambrano, C., Rojas, D., Carvajal, K., y Acuña, G. (2011). Análisis de rendimiento académico estudiantil usando data warehouse y redes neuronales. Ingeniare. Revista chilena de ingeniería, 19(3), 369-381. https://doi.org/10.4067/S0718-33052011000300007 